\title{
Elevated interleukin-2 receptor level in patients with active pulmonary tuberculosis and the changes following anti-tuberculosis chemotherapy
}

\author{
C.H.S. Chan, C.K.W. Lai, J.C.K. Leung, A.S.S. Ho, K.N. Lai
}

Elevated interleukin-2 receptor level in patients with active pulmonary tuberculosis and the changes following anti-tuberculosis chemotherapy. C.H.S. Chan, C.K.W. Lai, J.C.K. Leung, A.S.S. Ho, K.N. Lai. CERS Journals Ltd 1995.

ABSTRACT: Soluble interleukin-2 receptor (SIL-2R) is a marker of T-lymphocyte activation. We have undertaken a study to examine the serum sIL-2R levels in patients with pulmonary tuberculosis (TB) and the changes following anti-TB chemotherapy.

Forty four patients with pulmonary TB or tuberculous pleural effusion were recruited. Serum was collected from the patients before and at 1, 2, 4 and 6 months after initiation of anti-TB chemotherapy. Serum sIL-2R level was measured by an enzyme immunoassay.

The mean sIL-2R level before treatment was $1,452 \pm 103$ (SEM) $\mathrm{U}^{\cdot} \mathrm{ml}^{-1}$, which was significantly higher than that of healthy control subjects $\left(374 \pm 30 \mathrm{U} \cdot \mathrm{ml}^{-1}\right)$. There was no significant change in the sIL-2R level at 1 month, but there was a gradual reduction from the second month onwards. At the sixth month the mean sIL-2R level was $1080 \pm 81 \mathrm{U} \cdot \mathrm{ml}^{-1}$, which was significantly lower than that before treatment. However, despite clinical improvement, the sIL-2R levels at the sixth month were still significantly higher than those of control subjects.

We conclude that sIL-2R levels were elevated in patients with pulmonary TB and there was a gradual reduction following anti-TB chemotherapy. However, the sIL-2R levels were still higher than control subjects at completion of treatment, suggesting a delayed resolution of the inflammation in patients with pulmonary TB.

Eur Respir J., 1995, 8, 70-73.
Dept of Medicine, Chinese University of Hong Kong, Prince of Wales Hospital, Shatin, NT, Hong Kong.

\author{
Correspondence: C.H.S. Chan \\ Dept of Medicine \\ Chinese University of Hong Kong \\ Prince of Wales Hospital \\ Shatin \\ NT \\ Hong Kong
}

Keywords: Chemotherapy

soluble interleukin-2 receptor

tuberculosis

Received: May 161994

Accepted after revision October 161994

This study was supported by UPGC small project grant A/C No. 22401420.
It has long been recognized that T-lymphocytes are essential in the immunity against tuberculosis (TB) [1$3]$. The interaction of mycobacteria with alveolar macrophages leads to activation of T-lymphocytes. As a result, interleukin-2 receptor (IL-2R) molecules are expressed on their cell surface [4] and soluble IL-2R (sIL-2R) molecules are released into the circulation [5].

As this soluble receptor retains some of the biological activities of the cell-associated IL-2R molecule, including its capacity to bind IL-2 efficiently [6], it is possible that sIL-2R could play a regulatory role in the immune response. Although the exact immunological role of sIL-2R is not well-established, it may serve as a marker of disease activity in patients with systemic lupus erythematosus, rheumatoid arthritis [7], haematological malignancies [8] and pulmonary disorders, such as asthma [9], lung cancer [10] and tuberculosis (TB) [11]. In patients with old inactive TB, the serum sIL-2R levels were not significantly different from those of normal control subjects. In contrast, active pulmonary TB is associated with markedly elevated sIL2R levels [11].

The effect of anti-TB chemotherapy on the cellular immune response is unclear. It has been suggested by some studies that anti-TB drugs may have an immunosuppressive effect [12], which may be reflected in the SIL-2R levels. We have undertaken a study of the sIL$2 \mathrm{R}$ levels in patients with pulmonary $\mathrm{TB}$ and have investigated their serial changes following treatment, to find out whether sIL-2R may serve as a marker of disease activity and the immune response following antiTB chemotherapy.

\section{Patients and methods}

Forty four consecutive patients (31 males and $13 \mathrm{fe}$ males) with newly diagnosed active pulmonary TB were recruited into the study. Their mean age was 57 yrs (range 16-89 yrs). Thirty three of them had pulmonary parenchymal lesion and 11 had pleural effusion. The diagnosis of TB was confirmed by positive smear and/or culture of tubercle bacilli in sputum, or the presence of caseating granulomata on pleural biopsy specimens. None of the patients had clinical evidence of the acquired immunodeficiency syndrome and none 
were on corticosteroids or other immunosuppressive agents. Patients with co-existing connective tissue disorders, haematological malignancies, and pulmonary diseases, such as asthma and lung cancer, were excluded from the study.

Blood samples were taken from the patients before initiation of anti-TB treatment and after informed consent was obtained. Blood samples taken from 41 healthy volunteers (17 males and 24 females) with a mean age of 31 yrs (range 21-47 yrs) were used as controls. Serum was separated from the clotted whole blood after centrifugation at $2,000 \times \mathrm{g}$ for $15 \mathrm{~min}$ and stored at $-70^{\circ} \mathrm{C}$ before sIL-2R assay. The patients were then treated with standard anti-TB drugs consisting of isoniazid $(300 \mathrm{mg}$ q.d.), rifampicin (450-600 mg q.d.), pyrazinamide (1.5$2 \mathrm{~g}$ q.d.) and ethambutol $\left(15 \mathrm{mg} \cdot \mathrm{kg}^{-1}\right.$ q.d.) for two months followed by isoniazid and rifampicin for four months [13]. During each follow-up visit at monthly intervals, the patients were assessed for their symptoms, and sputum specimens were sent for mycobacterial culture. Chest radiographs were taken at roughly two monthly intervals. After completion of anti-TB treatment for 6 months, the patients were then reassessed at three monthly intervals up to one year. A patient was considered cured if he had negative mycobacterial cutures on three separate occasions, together with resolution of symptoms and signs of healing on chest radiograph. Blood samples were again taken at 1, 2, 4 and 6 months after initiation of anti-TB treatment. All serum samples were randomly assigned code numbers, and assayed in a single batch without the knowledge of the clinical status or stage of treatment of the patients.

Serum sIL-2R level was measured by a commercial enzyme immunoassay (T-Cell Diagnostics, Cambridge, MA, USA) as described previously [9], using a sandwich technique with two different monoclonal antibodies that recognize different epitopes of the sIL-2R. sIL-2R values are expressed in units per $\mathrm{ml}\left(\mathrm{U} \cdot \mathrm{ml}^{-1}\right)$; one unit per $\mathrm{ml}$ sIL-2R is equivalent to $8.85 \mathrm{pg}$ per $\mathrm{ml}$ sIL2R. The detection limit of the kit is $24 \mathrm{U} \cdot \mathrm{ml}^{-1} \mathrm{sIL}-$ $2 \mathrm{R}$. All samples were assayed within a single batch and the intra-assay precision is $6.5 \%$.

\section{Data analysis}

All data are expressed as mean \pm SEM unless otherwise stated, and the $\mathrm{p}<0.05$ degree of significance was accepted. Group data on the sIL-2R levels were compared using Mann-Whitney test. Serial changes in sIL-2R levels were examined by analysis of variance (ANOVA), and any significance was further sought by least significance difference.

\section{Results}

The serum sIL-2R levels of the patients before and after anti-TB treatment are shown in figure 1 . The sIL-2R levels before treatment $\left(1,452 \pm 103 \mathrm{U} \cdot \mathrm{ml}^{-1}\right)$ were markedly raised compared to control subjects $\left(374 \pm 30 \mathrm{U} \cdot \mathrm{ml}^{-1}\right)$. After anti-TB chemotherapy there was a progressive

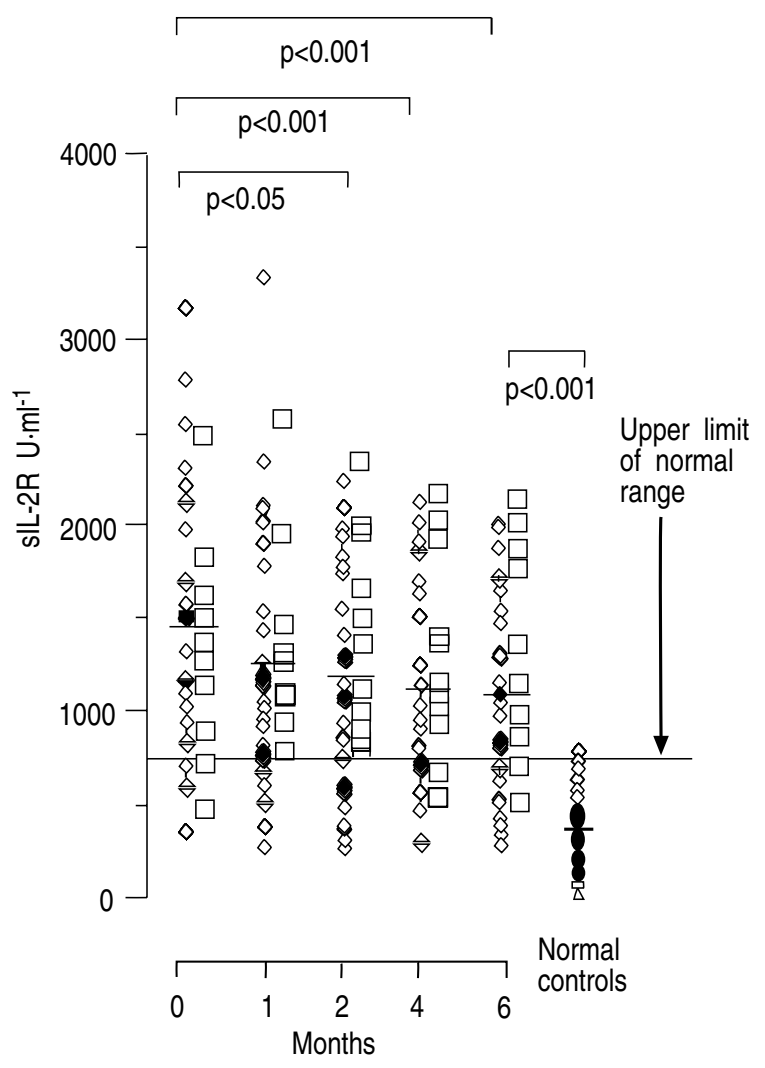

Fig. 1. - Changes in soluble interleukin-2 receptor (sIL-2R) level following anti-tuberculosis (TB) chemotherapy. $\Delta$ : normal; $\diamond$ : tuberculosis involving lung parenchyma; $\square$ : tuberculous pleural effusion. Horizontal bars indicate mean value.

reduction in the sIL-2R levels. The sIL-2R levels at 1 ,

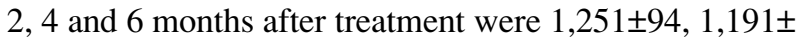
$83,1,118 \pm 85$ and $1,080 \pm 81 \mathrm{U} \cdot \mathrm{ml}^{-1}$, respectively. There was no significant difference between the sIL-2R levels before and at 1 month after anti-TB chemotherapy. From the second month onwards, there was a gradual decline in the sIL-2R levels, which were significantly lower than those before treatment. All except two patients had sputum culture negative from the first month onwards. These two patients were culture negative from the second month onwards. For these two cases, the sIL-2R levels showed a gradual decline similar to the other cases (Case No. 1: 1,173, 1,032, 853, 901 and 841 $\mathrm{U} \cdot \mathrm{ml}^{-1}$ at $0,1,2,4$ and 6 months, respectively; Case No. 2: 1,491, 1,209, 953, 714 and $162 \mathrm{U} \cdot \mathrm{ml}^{-1}$ at 0,1 , 2, 4 and 6 months, respectively).

After completion of anti-TB chemotherapy at 6 months, all the patients were clinically cured and none of the patients had persistence of mycobacteria in their sputum. However, the sIL-2R levels at the sixth month were still significantly higher than those of control subjects $(\mathrm{p}<0.001)$. If we define the normal range of serum sIL$2 \mathrm{R}$ value as mean $+2 \mathrm{SD}\left(\right.$ i.e. $758 \mathrm{U} \cdot \mathrm{ml}^{-1}$ ), then 40 patients $(91 \%)$ had raised sIL-2R levels before treatment. At completion of anti-TB chemotherapy after 6 months, 31 patients $(70 \%)$ still had raised sIL-2R levels. If we divide the patients into two groups: 1) patients with 
parenchymal lesions; and 2) patients with tuberculous pleural effusion (fig. 1), there was no significant difference in the sIL-2R levels between the two groups throughout the course of treatment.

\section{Discussion}

T-lymphocytes have been shown to play an important role in the immunity against mycobacterial infections [1-3]. This is confirmed by studies which show that bronchoalveolar lavage fluids from patients with active TB revealed more T-cells than control subjects [14, 15]. The activated T-lymphocytes produce lymphokines that help to prevent macrophages from leaving the site of inflammation, induce fusion and giant cell formation, chemotaxis and enhanced killing by macrophages [1]. As a result of mycobacterial infection and the subsequent T-lymphocyte activation, sIL-2R molecules are released into the circulation [5].

Although IL-2R are also expressed on B-cells and monocytes, the majority of cells bearing IL-2R are Tlymphocytes [16]. Therefore, the serum levels of sIL$2 \mathrm{R}$ may be used as a serological indicator of T-lymphocyte activation. In patients with active $\mathrm{TB}$, the cell lines producing sIL-2R is not certain. Because IL-2R was detected predominantly on T-lymphocytes after stimulation by purified protein derivative (PPD) [17], we have reason to believe that T-cells are the major source of sIL-2R in patients with active TB. However, an in vitro study has demonstrated the expression of IL-2R by peripheral blood monocytes from patients with active pulmonary TB [18]. Therefore, monocytes may contribute to the pool of circulating sIL-2R in patients with active TB. Further studies to evaluate the cell lines producing SIL-2R in patients with TB are warranted. Since activation and proliferation of T-lymphocytes and monocytes are associated with the expression of IL- $2 \mathrm{R}$ and subsequent release of sIL-2R, an elevated sIL-2R level in patients with TB provides a good marker of ongoing cell activation, and, thus, may be useful in evaluating the disease phase and the response to treatment.

The effect of anti-TB chemotherapy on the immune system is controversial. Some studies have demonstrated that anti-TB chemotherapy has no effect on immune response [19], whilst others have suggested that cell-mediated immunity is inhibited [12]. Serial measurements of serum sIL-2R level may be another way to assess the effect of anti-TB chemotherapy on the immune responsiveness in patients with pulmonary TB. It may also be interesting to evaluate if different drug regimens could have different or similar effect on T-lymphocyte and monocyte activation by serial sIL-2R measurements. In one study, the sIL-2R levels were persistently raised up to three months after initiation of anti-TB chemotherapy [20].

In our present study, there was no significant change between the levels before and at one month after treatment. However, from the second month onwards, there was a gradual but significant decline in the serum sIL$2 \mathrm{R}$ levels. At the completion of anti-TB chemotherapy for six months, the sIL-2R levels were significantly lower than those before treatment $(1,080 \pm 81$ vs $1,452 \pm$ $\left.103 \mathrm{U} \cdot \mathrm{ml}^{-1}, \mathrm{p}<0.001\right)$, although the levels at six months were still higher than those of control subjects (374 \pm $\left.30 \mathrm{U} \cdot \mathrm{ml}^{-1}, \mathrm{p}<0.001\right)$.

In patients with bacterial infections the sIL-2R levels rapidly come down with antibiotic treatment [21], and in patients with asthma the sIL-2R levels decline with steroid treatment [9]. Therefore, the prolonged elevation of sIL-2R in patients with pulmonary TB is unlikely to be due to long half-life of sIL-2R. It is more likely to be the result of prolonged antigenic stimulation and the subsequent activation of the T-lymphocytes and monocytes.

Serial measurements of sIL-2R level may be used to monitor the response to treatment. This is particularly useful when anti-TB chemotherapy has been started empirically on clinical or radiological grounds alone. A gradual decline in sIL-2R level is consistent with mycobacterial infection, whilst a rapid decline in sIL-2R level is more suggestive of bacterial infection. Failure to respond to treatment will be indicated by a persistent rise in the sIL-2R levels. In these cases, further investigations are indicated to make a definitive diagnosis rather than to continue empirical treatment. Furthermore, in patients with tuberculous lesions on chest radiographs with questionable activity, the sIL-2R level may be a further indicator to aid the decision on initiation of treatment. A normal sIL-2R level is suggestive of inactive disease and vice versa.

The gradual decline of sIL-2R levels in our patients from the second month onwards is more likely to be the result of clinical improvement than an inhibition of cellular immunity by anti-TB chemotherapy. However, the sIL-2R levels were still higher than control subjects at the sixth month, suggesting a delay in the resolution of pulmonary inflammation despite clinical improvement. Other studies have also demonstrated increased levels of inflammatory markers, such as circulating vascular cell adhesion molecule (cVCAM-1) in patients who had recently completed anti-TB treatment [22]. Another study has shown persistent lymphocytic alveolitis in patients with miliary TB at the end of anti-TB treatment [23]. In our previous study, we found that in patients with pulmonary TB treated over 12 months before, the sIL-2R levels were not different from that of normal [11], therefore we can extrapolate that the sIL-2R levels will slowly decline from the end of treatment down to the normal range after a few months.

The role of the elevated sIL-2R in the immunity against TB is uncertain. It could indicate an inappropriate activation of T-lymphocytes and macrophages/ monocytes, which may have harmful consequences. The perpetuation of the inflammatory response and lymphocytic alveolitis in the lungs of patients with pulmonary TB may lead to impairment of gaseous exchange. Cytokines derived from T-cells can have adverse physiological effects, including high fever, nausea, diarrhoea, hypertension, pulmonary oedema and endothelial leakage [24]. The beneficial effects of corticosteroids in some cases of TB $[25,26]$ may be mediated through the modulation of T-cell function. This may also explain the 
potential effect of corticosteroids in reducing the mortality during treatment in some cases of TB [27].

On the other hand, a prolonged elevation of sIL-2R reflecting a continued activation of T-lymphocytes and monocytes/macrophages may have a possible protective role. Living mycobacteria are typical long-lasting parasites within tuberculous lesions, and their antigenic components could be presented by macrophages to CD4 specific T-lymphocytes. The prolonged activation of $\mathrm{T}$ lymphocytes and monocytes/macrophages may have important protective roles against the long-lasting mycobacterial infection. The role played by sIL-2R in the inflammation could be different in the early phases (many actively growing mycobacteria, high antigenic load) or in the late phases during chemotherapy (low antigenic load). In addition different subsets of T-lymphocytes could be activated and could proliferate during early and late phases of immune response to mycobacterial antigens [28], so that the same elevation of sIL-2R may indicate activation of different T-lymphocyte subsets with different immunological roles.

In conclusion, we have found that serum sIL-2R levels were markedly elevated in patients with active pulmonary TB and that there was a gradual decline from the second month after initiation of anti-TB chemotherapy. However, the sIL-2R levels were still significantly higher than control subjects after six months of treatment, indicating a delay in the resolution of the inflammatory response despite successful anti-TB chemotherapy. The role of the prolonged elevation of sIL-2R in patients with TB awaits further investigation.

\section{References}

1. Edwards D, Kirkpatrick CH. The immunology of mycobacterial diseases. Am Rev Respir Dis 1986; 134: 1062-1071.

2. Ellner JJ, Wallis RS. Immunologic aspects of mycobacterial infections. Rev Infect Dis 1989; 11: S455-S459.

3. Maurice JL. Delayed hypersensitivity and immunity in tuberculosis. Am Rev Respir Dis 1975; 111: 243-246.

4. Cantrell DA, Smith KA. Transient expression of interleukin-2 receptors: consequences for T-cell growth. $J$ Exp Med 1983; 158: 1895-1911.

5. Rubin LA, Kurman CC, Fritz ME, et al. Soluble interleukin- 2 receptors are released from activated human lymphoid cells in vitro. J Immunol 1985; 135 : 3172-3177.

6. Rubin LA, Jay G, Nelson DL. The released interleukin2 receptor binds interleukin-2 efficiently. J Immunol 1986; 137: 3841-3844.

7. Semenzato G, Bambara LM, Biasi D, et al. Increased serum levels of soluble interleukin-2 receptor in patients with systemic lupus erythematosus and rheumatoid arthritis. J Clin Immunol 1988; 8: 447-452.

8. Chilosi M, Semenzato G, Vinante F, et al. Increased levels of soluble interleukin-2 receptor in non-Hodgkin's lymphoma. Am J Clin Pathol 1989; 92: 186-191.

9. Lai CKW, Chan CHS, Leung JCK, Lai KN. Serum concentration of soluble interleukin-2 receptors in asthma: correlation with disease activity. Chest $1993 ; 103$ : 782-786.

10. Chan CHS, Ho J, Lai CKW, Leung JCK, Lai KN.
Elevated serum levels of soluble interleukin-2 receptors in lung cancer and the effect of surgery. Respir Med 1993; 87: 383-385.

11. Chan CHS, Lai KN, Leung JCK, Lai CKW. T-lymphocyte activation in patients with active tuberculosis. Am Rev Respir Dis 1991; 144: 458-460.

12. Ruben FL, Winkelstein A, Fotiadis IG. Immunological responsiveness of tuberculosis patients receiving rifampin. Antimicrob Agents Chemother 1974; 5: 383-387.

13. Ormerod LP. Chemotherapy and management of tuberculosis in the United Kingdom: recommendations of the Joint Tuberculosis Committee of the British Thoracic Society. Thorax 1990; 45: 403-408.

14. Dhand R, De A, Ganguly NK, et al. Factors influencing the cellular response in bronchoalveolar lavage and peripheral blood of patients with pulmonary tuberculosis. Tubercle 1988; 69: 161-173.

15. Hoheisel GB, Tabak L, Teschler H, Erkan F, Kroegel C, Costabel U. Bronchoalveolar lavage cytology and immunocytology in pulmonary tuberculosis. Am J Respir Crit Care Med 1994; 149: 460-463.

16. Nelson DL, Rubin LA, Kurman CC, Fritz ME, Boutin B. An analysis of the cellular requirements for the production of soluble interleukin-2 receptor in vivo. J Clin Immunol 1986; 6: 114-120.

17. Platt JL, Grant BW, Eddy AA, Michael AF. Immune cell populations in cutaneous delayed-type hypersensitivity. J Exp Med 1983; 158: 1227-1242.

18. Toosi Z, Zedor JR, Lapurga JP, Ondash RJ, Ellner JJ. Expression of functional interleukin-2 receptors by peripheral blood monocytes from patients with active pulmonary tuberculosis. J Clin Invest 1990; 85: 1777-1784.

19. Humber DP, Nsanzumuhire H, Aluoch JA, et al. Controlled double-blind study of the effect of rifampicin on humoral and cellular immune responses in patients with pulmonary tuberculosis and in tuberculosis contacts. Am Rev Respir Dis 1980; 122: 425-436.

20. Brown AE, Rieder KT, Webster HK. Prolonged elevation of soluble interleukin-2 receptors in tuberculosis. Am Rev Respir Dis 1989; 139: 1036-1038.

21. Lawrence EC, Holland VA, Young JB, et al. Dynamic changes in soluble interleukin-2 receptor levels after lung or heart-lung transplantation. Am Rev Respir Dis 1989; 140: 789-796.

22. Lai CKW, Wong KC, Chan CHS, Ho SS, Chung SY, Haskard DO, Lai KN. Circulating adhesion molecules in tuberculosis. Clin Exp Immunol 1993; 94: 522-526.

23. Sharma SK, Pande JN, Singh YN, et al. Pulmonary function and immunologic abnormalities im miliary tuberculosis. Am Rev Respir Dis 1992; 145: 1167-1171.

24. Davies AJS, Wallis VJ, Morrison WI. The trouble with T-cells. Lancet 1990; 335: 1574-1576.

25. Johnson JR, Turk TL, MacDonald FM. Corticosteroids in pulmonary tuberculosis. III. Indications. Am Rev Respir Dis 1967; 96: 62-73.

26. Chan HS, Pang JA. Effect of corticosteroids on deterioration of endobronchial tuberculosis during chemotherapy. Chest 1989; 96: 1195-1196.

27. Ellis ME, Webb AK. Cause of death in patients admitted to hospital for pulmonary tuberculosis. Lancet 1983; i: 665-667.

28. Ainslie GM, Solomon JA, Bateman ED. Lymphocyte and lymphocyte subset numbers in blood and in bronchoalveolar lavage and pleural fluid in various forms of human pulmonary tuberculosis at presentation and during recovery. Thorax 1992; 47: 513-518. 